\title{
Quantification of Intracranial Aneurysm Volume Pulsation with 7T MRI
}

\author{
(D) R. Kleinloog, (D).J.M. Zwanenburg, (D) B. Schermers, (DE. Krikken, (D).M. Ruigrok, (DP.R. Luijten, (D). Visser, (D). Regli, (D).J.E. Rinkel, \\ and (DB.H. Verweij \\ स
}

\begin{abstract}
BACKGROUND AND PURPOSE: Aneurysm volume pulsation is a potential predictor of intracranial aneurysm rupture. We evaluated whether 7T MR imaging can quantify aneurysm volume pulsation.

MATERIALS AND METHODS: In Stage I of the study, 10 unruptured aneurysms in 9 patients were studied using a high-resolution (0.6-mm, isotropic) 3D gradient-echo sequence with cardiac gating. Semiautomatic segmentation was used to measure aneurysm volume (in cubic millimeters) per cardiac phase. Aneurysm pulsation was defined as the relative increase in volume between the phase with the smallest volume and the phase with the largest volume. The accuracy and precision of the measured volume pulsations were addressed by digital phantom simulations and a repeat image analysis. In Stage II, the imaging protocol was optimized and 9 patients with 9 aneurysms were studied with and without administration of a contrast agent.
\end{abstract}

RESULTS: The mean aneurysm pulsation in Stage I was 8\% $\pm 7 \%$ (range, 2\%-27\%), with a mean volume change of $15 \pm 14 \mathrm{~mm}^{3}$ (range, 3-51 $\mathrm{mm}^{3}$ ). The mean difference in volume change for the repeat image analysis was $2 \pm 6 \mathrm{~mm}^{3}$. The artifactual volume pulsations measured with the digital phantom simulations were of the same magnitude as the volume pulsations observed in the patient data, even after protocol optimization in Stage II.

CONCLUSIONS: Volume pulsation quantification with the current imaging protocol on 7T MR imaging is not accurate due to multiple imaging artifacts. Future studies should always include aneurysm-specific accuracy analysis.

ABBREVIATIONS: CNR = contrast-to-noise-ratio; $\mathrm{TFE}=$ turbo field echo

ntracranial aneurysms occur in approximately $3 \%$ of the population. ${ }^{1}$ Rupture of an aneurysm results in aneurysmal sub-

Received May 26, 2017; accepted after revision November 30.

From the Department of Neurology and Neurosurgery (R.K., B.S., E.K., Y.M.R., L.R., G.J.E.R., B.H.V.), Brain Center Rudolf Magnus, and Department of Radiology (J.J.M.Z. P.R.L., F.V.), Utrecht University, University Medical Center Utrecht, Utrecht, the Netherlands; Department of Technical Medicine (B.S., E.K.), Faculty of Science and Technology, University of Twente, Enschede, the Netherlands; Philips Healthcare (F.V.), Best, the Netherlands; and Department of Neurosurgery (L.R.), University Hospital Zurich, Zurich, Switzerland.

R. Kleinloog was supported by a Focus en massa cardiovascular research grant by the Utrecht University, the Netherlands. Y.M. Ruigrok was supported by a clinical fellowship grant from the Netherlands Organization for Scientific Research (Project No. 40-00703-98-13533). J.J.M. Zwanenburg was supported by the European Research Council under the European Union's Seventh Framework Programme (FP7/2007-2013)/European Research Council grant agreement No. 337333.

This article has not been submitted or published elsewhere in whole or in part, except as an abstract (poster at European Stroke Conference, May 28-31, 2013; London, United Kingdom; Vasospasm, July 10-12, 2013; Lucerne, Switzerland; and Joint Annual Meeting of the International Society for Magnetic Resonance in Medicine-European Society for Magnetic Resonance in Medicine and Biology, Milan, Italy; May 10-16, 2014).

Please address correspondence to Rachel Kleinloog, MD, Brain Center Rudolf Magnus, Department of Neurology and Neurosurgery, Room G.03.232, Utrecht University, University Medical Center Utrecht, Heidelberglaan 100, 3584 CX Utrecht, the Netherlands; e-mail: rachelkleinloog@gmail.com arachnoid hemorrhage, which often occurs at a younger age and has a high case fatality and morbidity. ${ }^{2}$ Current standard treatment consists of neurosurgical clipping or endovascular coiling and can prevent rupture but carries a $4 \%-8 \%$ risk of major complications, including death, depending on the age of the patient and the size and site of the aneurysm. ${ }^{3,4}$ Preventive treatment should therefore ideally be restricted to those patients who have a high risk of rupture. However, prediction of the risk of rupture of intracranial aneurysms is poor. Therefore, better predictors of rupture are needed. Volume pulsation, the change in volume during the cardiac cycle, is a potential predictor of rupture. ${ }^{5}$ Imaging techniques used thus far in attempts to visualize volume pulsation, such as $1.5 \mathrm{~T}$ phase-contrast MR angiography, transcranial Doppler sonography, 3D rotational angiography, and 4D CTA, have various limitations, including limited signal-to-noise ratio,

\footnotetext{
- Indicates open access to non-subscribers at www.ajnr.org

Indicates article with supplemental on-line tables and appendix.

Indicates article with supplemental on-line photos.

Indicates article with supplemental on-line video.

http://dx.doi.org/10.3174/ajnr.A5546
}

AJNR Am J Neuroradiol 39:713-19 Apr 2018 www.ajnr.org 
limited spatial resolution compared with the aneurysm volume, and/or the use of a radiation dose and/or the risk of complications. ${ }^{5}$ Furthermore, a test of the accuracy and precision of the volume pulsation measurement was either lacking or studies refrained from giving an error value that could be used to interpret the pulsation results for each aneurysm. Therefore, aneurysm volume pulsation is currently not used as a predictor of rupture in clinical practice.

In this experimental study, we evaluated whether volume pulsation could be quantified on images obtained with 7T MR imaging and tested the accuracy and precision of the method.

\section{MATERIALS AND METHODS}

Our study consisted of 2 stages. In the first stage, we tested the concept of quantification of aneurysm pulsation on images obtained with 7T MR imaging, and we tested the accuracy and repeatability of the imaging-analysis method. In the second stage, we implemented the lessons learned in Stage I to optimize the imaging protocol and accuracy of the quantification of aneurysm pulsation.

\section{Study Population}

Patients diagnosed with saccular intradural unruptured intracranial aneurysms who were either scheduled for treatment of their aneurysm or were in follow-up for growth of their aneurysm were recruited through our outpatient clinic of the Department of Neurology and Neurosurgery of the University Medical Center Utrecht, the Netherlands, between July 2011 and December 2012 as part of an existing study focusing on imaging of the aneurysm wall (Stage I). ${ }^{6}$ In Stage II, additional patients were recruited for the current study between February and April 2014. The diagnosis of the aneurysm was made either on CTA or 1.5T or 3T MRA, and the aneurysms were either incidental findings (the imaging study was for other indications), found during screening because of a positive family history of intracranial aneurysm, or symptomatic aneurysms (eg, leading to a seizure or thromboembolic event). Patients with contraindications for 7T MR imaging (eg, claustrophobia, metal objects such as dental implants or prostheses in or on the body) were excluded, as well as patients with aneurysms associated with vascular malformations other than aneurysms (eg, arteriovenous malformations). In the second stage, patients with renal insufficiency and allergy to gadolinium-based contrast agent were also excluded. This study was approved by the institutional review board of our center, and all participants gave written informed consent following guidelines equivalent to the National Institutes of Health guidelines.

\section{T MR Imaging}

Imaging was performed on a 7T MR imaging scanner (Achieva; Philips Healthcare, Best, the Netherlands) with a volume transmit coil and a 32-channel receive head coil (2TX/32RX; Nova Medical, Wilmington, Massachusetts). In the first stage, the volume pulsation of the aneurysm was studied by adding a $3 \mathrm{D}$ turbo field echo (TFE) sequence to an existing study focusing on imaging of the aneurysm wall. ${ }^{6}$ This protocol included a low-resolution T1weighted survey sequence and a TOF sequence covering the intracranial vessels from the level of the circle of Willis and upward, taking into account the location of the aneurysm. ${ }^{6}$ It also included a previously described time-resolved 3D phase-contrast MR imaging sequence. ${ }^{7}$ The following parameters were used in the $3 \mathrm{D}$ TFE sequence: $\mathrm{TR} / \mathrm{TE}=8.2 / 4.4 \mathrm{~ms}$, flip angle $=6^{\circ}, \mathrm{FOV}=180 \times$ $180 \times 9.6 \mathrm{~mm}$, acquired spatial resolution $=0.6 \times 0.6 \times 0.6 \mathrm{~mm}$, sensitivity encoding acceleration factor $=2.0$ (right-left), and acquired temporal resolution $=90 \mathrm{~ms}$ interpolated to 15 cardiac phases. The sequence was synchronized to the heart with a peripheral pulse unit and retrospective cardiac gating. The scan duration was approximately 4 minutes. The TFE sequence was oriented in either a coronal or transverse orientation, depending on the location of the aneurysm in the circle of Willis and its orientation, and carefully positioned over the aneurysm as observed on a TOF and/or low-resolution T1-weighted survey. In both the TOF and the TFE sequences, flow-compensated gradients were used to prevent signal drop-out from fast-flowing blood.

With the results of Stage I, the imaging protocol was optimized. An optimized version of the TFE sequence was developed and used in the additionally recruited patients in Stage II. We increased the FOV, thereby increasing the signal-to-noise ratio and improving coverage; we shortened the TE to $2.0 \mathrm{~ms}$ to decrease the influence of the flow-displacement artifacts; and we used a $20^{\circ}$ flip angle to increase the contrast-to-noise ratio (CNR) between flowing blood and static tissue. Furthermore, we performed this improved TFE sequence after administration of gadobutrol, a gadolinium-based contrast agent, to further increase the CNR and decrease the influence of intensity fluctuations due to inflow effects.

\section{Image Analysis}

Volume Pulsation Quantification. Image analysis was performed with Analyze 11.0 software (AnalyzeDirect, Overland Park, Kansas), designed to automatically segment and calculate volumes. For the cardiac phases of each slice (15 phases in the first stage of the study, 18 in the second stage), the same ROI containing the aneurysm was chosen on the TFE images, using the TOF or T1weighted survey image as a $3 \mathrm{D}$ reference to correctly separate the aneurysm from the parent artery. Vessel segmentation (including the aneurysm) was performed by setting a signal intensity threshold. The threshold was defined by R.K. by visual inspection of the aneurysm and its surroundings and after comparing the TFE images with the anatomy of the aneurysm on the TOF and/or lowresolution T1-weighted survey. The threshold was adjusted until the aneurysm was distinguishable from the background and at the same time had the same appearance as on the anatomic scan. The threshold was set for each aneurysm separately but was fixed for all slices and cardiac phases of each aneurysm. Thereafter, the remaining parent vessel was manually deselected (by R.K.) on each slice of the first phase of the cardiac cycle; then this deselected area was copied to the 14 (or 17 in Stage II) consecutive phases of the cardiac cycle and removed. Flow artifacts in the lumen of the aneurysm were misrecognized by the software and consequently manually included in the selection, keeping the borders of the aneurysm as selected by the software intact. Voxels selected outside the aneurysm borders due to background noise were also removed from the selection. The manual corrections were done slice by slice. If the signal in the last slice containing the aneurysm 
was of low intensity due to partial volume effects, it was sometimes difficult to distinguish noise pixels from aneurysm voxels. Consequently, such a slice was excluded in all phases to limit the effect of noise on the selection. For each phase of the cardiac cycle, the total volume of the aneurysm was calculated by adding up the aneurysm volumes of all slices (segmented area $\times$ slice thickness). Absolute volume pulsation was defined as the change in volume (in cubic millimeters) between the phase of the cardiac cycle with the smallest volume and the phase with the largest volume. Relative volume pulsation was calculated with the following formula: [(Maximum Volume - Minimum Volume)/Minimum Volume] $\times$ 100.

The size (largest diameter) of the aneurysms was measured on TOF angiography in the first stage. In the second stage, it was measured on the improved TFE. Partially thrombosed aneurysms were excluded from the image analysis.

Repeatability of the Volume Pulsation Quantification (Stage I). By repeating the volume measurements for the phases with the smallest and largest volumes for each aneurysm, we determined the precision of the pulsation analysis. The repeat analysis was performed by the same observer but blinded for the initial analysis with a 3-month interval between the 2 analyses. We evaluated the repeatability of the image analysis using the Bland-Altman method: The mean and SD of the difference between the results of the initial analysis and the results of the repeat analysis were calculated. ${ }^{8}$ A $t$ distribution with a significance level of .05 and $9 d f(10$ measured aneurysms) was used to approximate the distribution of the 95\% CI. In addition, the Pearson correlation coefficient between the initial analysis and the repeat analysis was calculated. The repeatability analysis was performed only in Stage I of this study.

Accuracy of the Volume Pulsation Quantification (Stage I). The accuracy of the volume pulsation analysis is expected to depend on 4 different factors: the CNR (between blood and surroundings) of the images, signal intensity fluctuations in the gated TFE sequence due to inflow effects, aneurysm size, and blood flow artifacts.

\section{CNR, Signal Intensity Fluctuations, and Aneurysm Size}

To estimate the influence of CNR, signal intensity fluctuations, and aneurysm size on the calculated volume pulsations, we performed digital phantom simulations in which the 3 factors that are thought to influence the accuracy were varied. See the On-line Appendix for further details. In these simulations, the CNR was defined as

$$
\mathrm{CNR}=\frac{\text { Contrast }}{\text { Noise }}=\frac{\mu_{\mathrm{s}}-\mu_{\mathrm{bg}}}{\sigma_{\mathrm{s}}},
$$

with $\mu_{\mathrm{s}}$ as the mean pixel value in the phantom region, and $\mu_{\mathrm{bg}}$ as the mean pixel value in a background region, both averaged across the time phases in the cardiac cycle (15 in the first stage of the study and 18 in the second stage). $\sigma_{\mathrm{s}}$ is the mean of the time phase $\mathrm{SD}$ of each pixel in the signal phantom region. In the first digital phantom experiment, the dependence of the inaccuracy of the volume pulsation analysis of both CNR and signal fluctuations was studied. Second, the interaction of aneurysm volume with the
CNR and signal fluctuations was studied by static digital phantom simulations. Third, aneurysm-specific inaccuracy in the pulsation analysis was measured for each aneurysm in the patient study by making a pulsating digital phantom with the same volume, CNR, intensity fluctuation, and volume pulsation. The difference in the volume pulsation measured in the digital phantoms and the volume pulsation measured in the patient scans (and used as input for the phantom volume pulsation) was called the "absolute observed artifactual pulsation.”

\section{Flow-Displacement Artifacts}

In image acquisition, the timing difference between the moment of phase-encoding (right after excitation) and the moment of frequency-encoding (approximately at $\mathrm{t}=\mathrm{TE}$ ) will lead to the flowdisplacement artifacts. ${ }^{9}$ We assessed the potential influence of these artifacts by combining the TFE images from Stage I with images containing blood flow velocity data (obtained with the time-resolved $3 \mathrm{D}$ phase-contrast $\mathrm{MR}$ imaging sequence, only available in Stage I; see the On-line Appendix for further details).

Imaging Protocol Optimization (Stage II). Volume pulsation quantification on images obtained with the improved TFE was analyzed with the same analysis tool used in Stage I. As in Stage I, the accuracy of the volume pulsation analysis method was addressed in the pulsating phantoms. For both the improved TFE and the gadolinium-enhanced improved TFE patient data, pulsating phantoms were made to estimate the (aneurysm-specific) inaccuracy in the pulsation. The volume pulsation measurement in the phantom was compared with the given volume pulsation. This difference yielded the absolute observed artifactual pulsation.

\section{RESULTS}

An overview of the results of Stages I and II are given in the Table.

\section{Stage I: Volume Pulsation Quantification, Repeatability, and Accuracy of the Analysis}

In Stage I, the mean aneurysm volume change was $15 \pm 14 \mathrm{~mm}^{3}$ (range, $3-51 \mathrm{~mm}^{3}$ ), and the mean volume pulsation was $8 \% \pm 7 \%$ (range, 2\%-27\%). The mean time between the minimum and maximum volume was 7 phases (range, $2-12$ phases). The timing of the minimum and maximum volumes within the cardiac cycle was inconsistent among different patients, with the minimum volume followed by the maximum volume in some patients, while the maximum was followed by the minimum volume in others (On-line Table 1). Fig 1 shows the 15 images obtained during the cardiac cycle of 1 cross-section of an aneurysm, and this pulsating aneurysm is provided as an On-line Video. The repeatability analysis yielded a correlation coefficient of 0.95 (Fig 2). The mean of the difference between the results of the initial pulsation analysis and the results of the repeat pulsation analysis was $2 \pm 6 \mathrm{~mm}^{3}$ (95\% CI, - 12-15; Fig 2).

The absolute observed pulsation in a static digital phantom (used as a measure of the inaccuracy of the analysis) was found to increase quickly below a CNR of approximately 6 and increased with increasing signal intensity fluctuations (see the On-line Ap- 


\begin{tabular}{lccc} 
& & \multicolumn{2}{c}{ Stage II } \\
\cline { 2 - 4 } \multicolumn{1}{c}{ Sequence } & Stage I TFE & Improved TFE & CE-Improved TFE \\
\hline No. of phases in the cardiac cycle & 15 & 18 & 18 \\
No. of patients included & 9 & 9 & $8^{\text {a }}$ \\
No. of aneurysms included & 10 & 9 & $8^{\mathrm{a}}$ \\
Mean size of the aneurysms (SD, range) $(\mathrm{mm})$ & $9(4,3-19)$ & $8(4,2-14)$ & $8(4,2-14)$ \\
Mean aneurysm volume change (SD, range) $\left(\mathrm{mm}^{3}\right)$ & $15(14,3-51)$ & $38(31,2-88)$ & $14(9,1-25)$ \\
Mean volume pulsation (SD, range) $(\%)$ & $8(7,2-27)$ & $39(24,14-73)$ & $15(11,4-36)$ \\
Mean absolute observed artifactual pulsation (SD, range) $\left(\mathrm{mm}^{3}\right)$ & $2(2,0-5)$ & - & $13(9,1-27)$ \\
\hline
\end{tabular}

Note:-CE indicates contrast-enhanced.

${ }^{a}$ In 1 patient, the image acquisition of the contrast-enhanced-improved TFE failed.

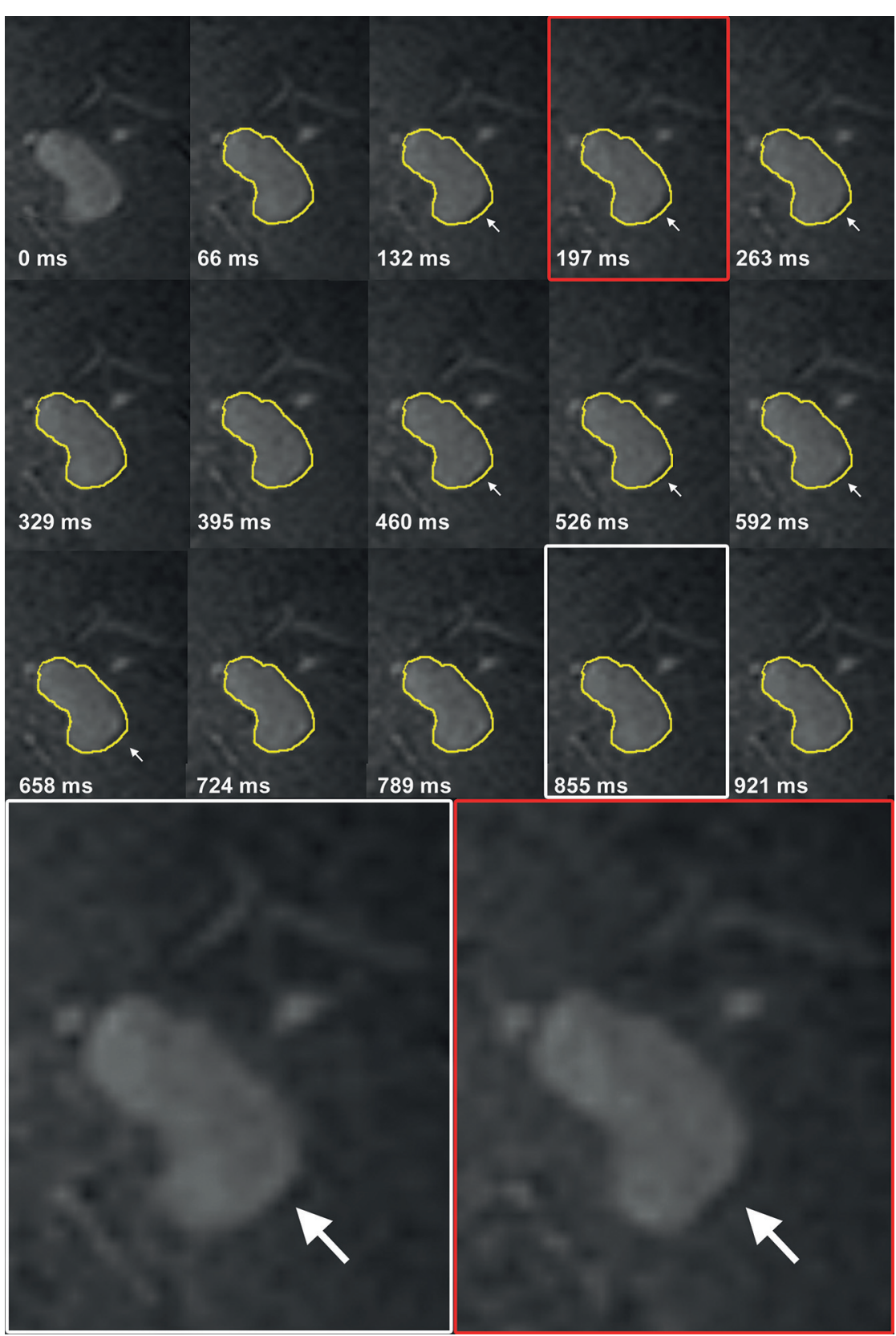

FIG 1. Coronal cross-section of a left middle cerebral artery aneurysm for each of the 15 phases of the cardiac cycle (heart rate, 60 beats per minute). The contour of the aneurysm at $0 \mathrm{~ms}$ is shown in yellow on all other time points. The white arrows indicate the area of pulsation in this cross-section. Magnification of 2 phases shows the deformation of the aneurysm at 1 side of the dome. pendix for further details and figures). The mean CNR of aneurysms in the patient study was 5 (range, 1-8), while the mean intensity fluctuation was 3\% $1.6 \%$. Both the effects of the CNR and intensity fluctuations were size-dependent, with increasing relative inaccuracy with decreasing phantom volumes. The mean absolute observed artifactual pulsation in the pulsating digital phantoms (used as a measure for the inaccuracy of the analysis) was $2.0 \pm 1.6 \mathrm{~mm}^{3}$ (range, 0.2-5.4 $\mathrm{mm}^{3}$; On-line Tables 1 and 2). The potential pulsation observed as a result of the flow displacement artifacts depended considerably on the orientation of the acquisition. The worst case artifactual pulsation was equal to or higher than the actual pulsation measured in 4 of the 6 available patient datasets (On-line Table 3). The minimum artifactual volume pulsation was around $25 \%$ of the volume pulsation measured in the patient scans.

\section{Stage II: Results after Imaging Protocol Optimization}

The mean aneurysm volume change on the improved TFE was $38 \pm 31 \mathrm{~mm}^{3}$ (range, 2-88 $\mathrm{mm}^{3}$ ), and the mean volume pulsation was $39 \% \pm 24 \%$ (range, $14 \%-73 \%)$. The mean aneurysm volume change on the contrast-enhanced improved TFE was $14 \pm 9 \mathrm{~mm}^{3}$ (range, 1-25 $\mathrm{mm}^{3}$ ), and the mean volume pulsation was $15 \% \pm 11 \%$ (range, $4 \%-$ $36 \%$ ). The mean time between the minimum and the maximum volume was 8 phases (range, 3-14 phases) in the improved TFE and 7 phases (range, 3-13 phases) in the contrast-enhanced TFE. As in Stage I, the timing of the minimum and maximum volumes within the cardiac cycle differed among patients (Online Table 4). The mean CNR was $23 \pm 7$ 

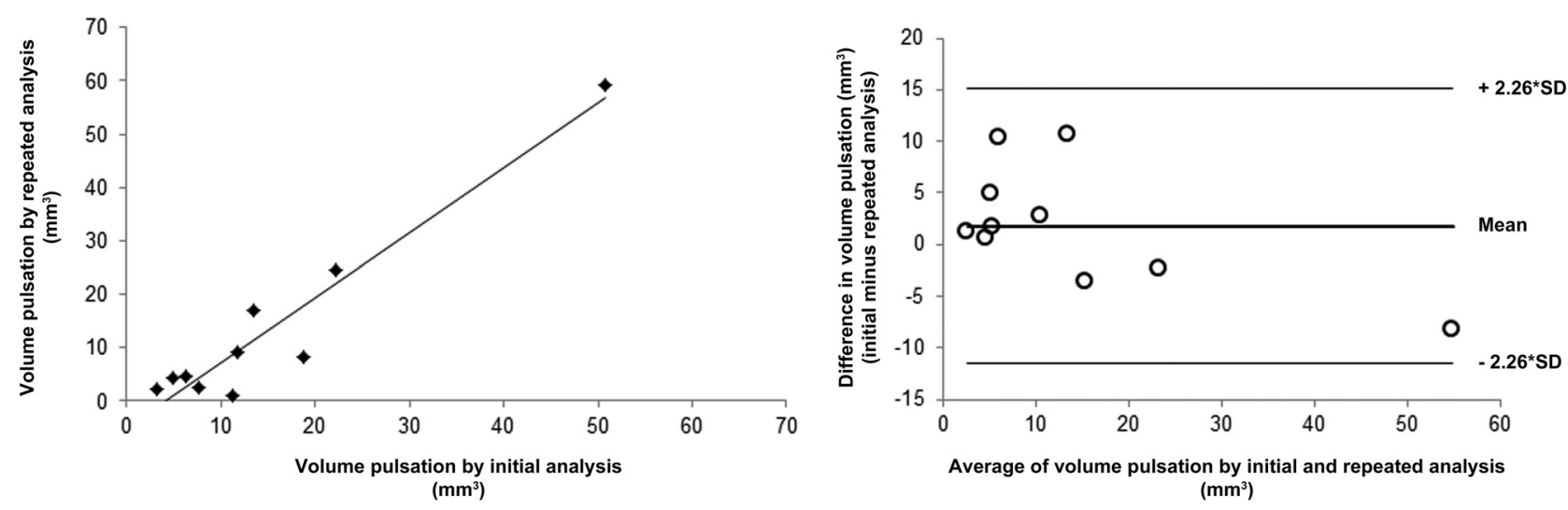

FIG 2. Repeatability of the image analysis method. Left: scatterplot showing the correlation between the measured volume pulsation obtained with the repeat image analysis versus the results from the initial analysis. Right: Bland-Altman plot of the same data.

in the improved TFE and $28 \pm 8$ in the gadolinium-enhanced improved TFE, while the mean intensity fluctuation was $23 \%$ $\pm 7 \%$ in the improved TFE and $15 \% \pm 6 \%$ in the gadoliniumenhanced improved TFE. The mean absolute observed artifactual pulsation in the pulsating digital phantoms (used as a measure of the inaccuracy of the analysis) was $13 \pm 9 \mathrm{~mm}^{3}$ (range, 1-27 $\mathrm{mm}^{3}$; On-line Tables 4 and 5).

\section{DISCUSSION}

This study shows that quantification of volume pulsation of unruptured intracranial aneurysms is currently not feasible using a semiautomatic segmentation method, even on highresolution 7T MR images. The accuracy of the quantification of volume pulsation is influenced by the CNR, intensity fluctuations, aneurysm size, and, most important, the flow-displacement artifacts. Our study shows that the artifactual volume pulsation due to these influences is of the same magnitude as the volume pulsations observed in the patient data, even after optimization of the imaging sequences and adding contrast agent.

Three previous studies quantified pulsation in unruptured aneurysms using an electrocardiography-gated CTA. ${ }^{10-12}$ Two of these studies found a volume pulsation in the range of $3 \%-18 \%$ with a mean of $8 \% \pm 5 \%,{ }^{10}$ and in the range of $1 \%-15 \%$ with a mean of $5 \% \pm 4 \%,{ }^{11}$ which compares well with our findings. The third study did not report volume pulsations but focused on the feasibility of imaging pulsation by comparing the aneurysm volume curves and random curves with the arterial pulse wave (as a measure for "true" pulsation) and found that the aneurysm volume curves showed higher similarity to the pulse wave than the random curves but only in a subset of the aneurysms under study. ${ }^{12}$

Accuracy and precision analyses were performed in all CTA studies. The first study found a mean of $8 \%$ volume pulsation in aneurysms, which was considered substantial in comparison with a $2 \%$ volume change found in bone tissue. ${ }^{10}$ In the second study, a large, static phantom (a syringe filled with saline) was imaged to study the artificial volume pulsation, and it was concluded that its volume change of $<0.248 \%$ was an insignificant artifact. ${ }^{11} \mathrm{How}-$ ever, in the current study, we showed that inaccuracy increases with decreasing phantom size; therefore, their accuracy analysis might underestimate the artifactual pulsation because the phantom used was much larger than the aneurysms studied. The second study measured an interobserver discrepancy in the repeat measurement of 5 aneurysms of $11.9 \pm 17.6 \mathrm{~mm}^{3}$, which was considered acceptable, given a mean expansion volume of all aneurysms of $27.87 \pm 60.53 \mathrm{~mm}^{3}$. ${ }^{11}$ The third study found an intraand interobserver variability of around 0.5 of the postprocessing method used, which is fair. The first and second studies refrained from giving an error value that can be used to interpret the pulsation results for each aneurysm. Our study shows that the error is influenced by multiple artifacts and is aneurysm-specific, which makes it difficult to compare the accuracy of 7T MR imaging with the reported CTA accuracies. Furthermore, CTA has its own technique-specific artifacts, which should be taken into account when analyzing the accuracy of the pulsation measurement. ${ }^{5}$

We used different signal intensity-based thresholds in different aneurysms, without explicitly investigating the robustness of the aneurysm segmentation for various thresholds. Rather, we focused on the effect of signal intensity fluctuations, to give a more in-depth analysis of the behavior of the pulsation estimation as a function of the image characteristics. The segmentation depends on the actual CNR in combination with the signal intensity fluctuations. The threshold should be high enough to avoid including noise but not so high as to prevent exclusion of aneurysm pixels. If one ignored partial volume effects and assumed normally distributed noise, it is straightforward to analyze the sensitivity of the segmentation to the chosen threshold. If $<1 \%$ of the included pixels in the segmentation is allowed to be due to noise, the lower limit of the intensity threshold is approximately $2.5 \times$ $\mathrm{SD}$ (where SD = the SD of the noise). Increasing the threshold further would change the volume by $<1 \%$, provided that the threshold is not exceeding the upper limit where the aneurysm intensity will start failing to pass the threshold. Similarly, the upper limit would be $[(1-f) \times \mathrm{CNR}-2.5] \times \mathrm{SD}$, where $f$ is the intensity fluctuation as a fraction of the aneurysm signal (note that $\mathrm{CNR} \times \mathrm{SD}$ is the intensity of the aneurysm). From this simple reasoning, it would follow that a minimum CNR of 5 is required to allow robust segmentation without having too much noise, which matches the phantom simulations, which showed that the accuracy dropped quickly below a CNR of 6 .

Nonetheless, voxels with a partial volume effect should be con- 
sidered as well. They have an intensity somewhere in between the aneurysm intensity and the noise level, depending on the partial volume factor. Basically, the threshold determines how "full" a voxel should be to be counted as aneurysm voxel. In these voxels, intensity fluctuation is directly related to how many partial volume voxels will pass the intensity threshold. Thus, changing the threshold will lead to a change in (baseline) volume. However, because each partial volume fraction is equally likely to occur, the sensitivity to intensity fluctuations and to actual volume changes is not expected to depend on the chosen threshold.

This study has some limitations. First, we used a signal intensity-based threshold to segment the aneurysm volume but also showed that signal intensity fluctuations have a considerable influence on the accuracy of the volume pulsation analysis. Future studies should use an analysis method that is insensitive to signal intensity fluctuations throughout the cardiac cycle. For example, a recently published automatic segmentation method used for quantification and visualization of pulsations from electrocardiography-gated CTA data used the local deformation of image structures in all cardiac phases, which may be less dependent on the actual intensity. ${ }^{10}$ With such an automated method, the effects of manual correction are also eliminated and the precision of the measurement may be increased. Second, our estimate of aneurysm-specific inaccuracy was based on patient-specific measurements of CNRs and intensity fluctuations. Although care was taken to obtain representative values, one should note that both the CNR and intensity fluctuations may be variable along the border of the aneurysm. Also, the CNR measurements were likely influenced by intensity fluctuations. Because there was no separate noise acquisition in the MR imaging protocol, we used the SD of the ROI over the cardiac cycles, which may have led to an underestimation of the actual CNR (and, thus, to a slightly worse inaccuracy than actually present). Nonetheless, the accuracy analysis shows that aneurysm-specific inaccuracies should be determined and that an increase in the signal-to-noise ratio of the images should be achieved to improve the accuracy.

Third, our restricted FOV led to incomplete imaging of the largest aneurysm of $19 \mathrm{~mm}$ in Stage I of the study, and the subsequent pulsation only accounts for the imaged part. We were not able to expand the FOV due to a fixed scan protocol and therefore a fixed scan time. In the second stage of this study, we were able to increase the FOV to prevent this lack of coverage. Fourth, we found quite large differences in volume pulsation in Stage II of the study compared with Stage I. This finding may be a reflection of an actual difference among aneurysms because a different sample of patients was used in Stage II, which is not optimal when investigating an optimized imaging sequence. However, the most likely explanation is the substantial increase in signal intensity fluctuations in Stage II compared with Stage I, despite the aim of increasing the CNR and decreasing intensity fluctuations. Higher signal intensity pulsation leads to a decrease in volume pulsation quantification accuracy, and this is probably an important reason why a higher pulsation was measured in the aneurysms with the improved TFE in Stage II. Furthermore, the lower volume pulsation found in the same aneurysms on the contrast-enhanced TFE compared with the improved TFE can also be explained by lower signal intensity fluctuations in the contrast-enhanced TFE. Because lower signal intensity leads to increased accuracy of the volume pulsation quantification, the results of the contrast-enhanced TFE were considered more accurate than the results of the improved TFE. Nonetheless, the intensity pulsations with the contrast-enhanced TFE are still considerable (ranging from $7.5 \%$ to $24 \%$ ), which is still too high to make the volume pulsation analysis on this sequence accurate. Last, the clinical availability of 7T MR imaging is still limited but is expected to increase in the future.

The strength of this study is the use of an advanced highresolution imaging method to quantify pulsation in aneurysms, combined with a thorough accuracy analysis. All previous studies showed a single value for the inaccuracy, sometimes obtained from a large stable phantom. The phantom simulations in our study give insight into the relative contributions of different sources of measurement errors and their combined effect in a patient-specific way, which can stimulate future improvement on each of these aspects. An advantage of MR imaging over CTA is the lack of radiation in MR imaging. Furthermore, the ultra-high resolution of MR imaging combined with its ability to discriminate the aneurysm wall from its lumen provides a unique opportunity to search for new markers of rupture.

\section{CONCLUSIONS}

Quantification of intracranial aneurysm pulsation seemed to be feasible with 7T MR imaging, but even after optimization of the imaging protocol, the artifactual volume pulsations as a result of intensity fluctuations, the limited CNR, aneurysm size, and the flow displacement artifact have the same magnitude as the volume pulsations observed in the patient data. The current imaging protocol on 7T MR imaging is therefore of no use in studying pulsation as a risk factor for rupture at the moment. Future studies should always include an aneurysmspecific accuracy analysis.

\section{ACKNOWLEDGMENTS}

We acknowledge A. Zada and B. de Vries for their work on the accuracy analysis.

Disclosures: Rachel Kleinloog—RELATED: Grant: Focus en massa cardiovascular research grant by the Utrecht University, the Netherlands.* Jaco J. ZwanenburgUNRELATED: Grants/Grants Pending: European Research Council starting grant, Comments: Small-Vessel MRI, Grant No. 337333.* Fredy Visser-UNRELATED: Employment: Philips Healthcare. *Money paid to the institution.

\section{REFERENCES}

1. Vlak MH, Algra A, Brandenburg R, et al. Prevalence of unruptured intracranial aneurysms, with emphasis on sex, age, comorbidity, country, and time period: a systematic review and meta-analysis. Lancet Neurol 2011;10:626-36 CrossRef Medline

2. Nieuwkamp DJ, Setz LE, Algra A, et al. Changes in case fatality of aneurysmal subarachnoid haemorrhage over time, according to age, sex, and region: a meta-analysis. Lancet Neurol 2009;8:635-42 CrossRef Medline

3. Brinjikji W, Rabinstein AA, Nasr DM, et al. Better outcomes with treatment by coiling relative to clipping of unruptured intracranial aneurysms in the United States, 2001-2008. AJNR Am J Neuroradiol 2011;32:1071-75 CrossRef Medline

4. Naggara ON, Lecler A, Oppenheim C, et al. Endovascular treatment of intracranial unruptured aneurysms: a systematic review of the 
literature on safety with emphasis on subgroup analyses. Radiology 2012;263:828-35 CrossRef Medline

5. Vanrossomme AE, Eker OF, Thiran JP, et al. Intracranial aneurysms: wall motion analysis for prediction of rupture. AJNR Am J Neuroradiol 2015;36:1796-802 CrossRef Medline

6. Kleinloog R, Korkmaz E, Zwanenburg JJ, et al. Visualization of the aneurysm wall: a 7.0-Tesla magnetic resonance imaging study. Neurosurgery 2014;75:614-22; discussion 622 CrossRef Medline

7. van Ooij P, Zwanenburg JJ, Visser F, et al. Quantification and visualization of flow in the Circle of Willis: time-resolved three-dimensional phase contrast MRI at $7 \mathrm{~T}$ compared with $3 \mathrm{~T}$. Magn Reson Med 2013;69:868-76 CrossRef Medline

8. Bland JM, Altman DG. Applying the right statistics: analyses of measurement studies. Ultrasound Obstet Gynecol 2003;22:85-93 Medline
9. Larson TC 3rd, Kelly WM, Ehman RL, et al. Spatial misregistration of vascular flow during MR imaging of the CNS: cause and clinical significance. AJNR Am J Neuroradiol 1990;11:1041-48 Medline

10. Firouzian A, Manniesing R, Metz CT, et al. Quantification of intracranial aneurysm morphodynamics from ECG-gated CT angiography. Acad Radiol 2013;20:52-58 CrossRef Medline

11. Kuroda J, Kinoshita M, Tanaka H, et al. Cardiac cycle-related volume change in unruptured cerebral aneurysms: a detailed volume quantification study using 4-dimensional CT angiography. Stroke 2012;43:61-66 CrossRef Medline

12. Illies T, Saering D, Kinoshita M, et al. Feasibility of quantification of intracranial aneurysm pulsation with 4D CTA with manual and computer-aided post-processing. PLoS One 2016;11:e0166810 CrossRef Medline 